\title{
Transvaginal 3D Ultrasound Evaluation of Post-Cesarean Section Uterine Diverticulum
}

\author{
Cui-Lan Li ${ }^{*}$, Xue-Tang Mo1, Kai-Xuan Deng2, Dun-Jin Chen'1, Xing-Cheng Gao', \\ Jin-Guo Zhai ${ }^{1}$, Yi-Ming Liao', Hui-Ru Dai' ${ }^{1}$, Xiang Cai ${ }^{1}$ \\ ${ }^{1}$ The Third Affiliated Hospital of Guangzhou Medical University \& Key Laboratory for Major Obstetric Diseases \\ of Guangdong Province \& Key Laboratory of Reproduction and Genetics of Guangdong Higher Education \\ Institutes, Guangzhou, China \\ ${ }^{2}$ Department of Computer Science, Hong Kong Baptist University, Hong Kong, China \\ Email: ${ }^{*}$ cuilanli@gzhmu.edu.cn
}

Received 4 September 2015; accepted 12 October 2015; published 15 October 2015

Copyright @ 2015 by authors and Scientific Research Publishing Inc.

This work is licensed under the Creative Commons Attribution International License (CC BY). http://creativecommons.org/licenses/by/4.0/

(c) (i) Open Access

\begin{abstract}
Objectives: This study focused on evaluating the value of transvaginal 3D ultrasound for the diagnosis and prognostic assessment of post-cesarean section uterine diverticulum. Materials and Methods: We retrospectively analyzed the data from 32 patients with post-cesarean section uterine diverticulum over three recent years. In all patients, transvaginal 3D ultrasound was used to measure the size of the uterine diverticulum and the thickness of the lower uterine segment (LUS) and myometrium. Patients with a LUS with a myometrial thickness under $4 \mathrm{~mm}$ underwent resection and repair surgery; those with a LUS with a myometrial thickness over $4 \mathrm{~mm}$ underwent hysteroscopic resection. The postoperative sonograms were compared with preoperative images to evaluate the efficacy of various treatments. Results: The mean length, width and depth of the uterine diverticula were $18.30 \pm 2.80 \mathrm{~mm}, 9.14 \pm 3.20 \mathrm{~mm}$ and $11.49 \pm 2.71 \mathrm{~mm}$, respectively. The average LUS myometrial thickness was $3.40 \pm 0.80 \mathrm{~mm}$ (with a range of $1.6 \mathrm{~mm}-6.3 \mathrm{~mm}$ ). After surgery, two patients' sonograms still showed diverticula at the post-cesarean section scar, measuring $6 \mathrm{~mm} \times 7 \mathrm{~mm} \times 6 \mathrm{~mm}$ and $6 \mathrm{~mm} \times 8 \mathrm{~mm} \times 4 \mathrm{~mm}$. There were significant differences in the size of uterine diverticula between preoperative and postoperative sonograms $(P<0.05)$ and the effective rate of surgery was $93.75 \%(30 / 32)$. Conclusions: Transvaginal 3D ultrasound is an accurate method for detecting post-cesarean section uterine diverticulum and is helpful for assessing surgical options and prognosis. The LUS myometrial thickness, which is considered as an optional index of post-cesarean section uterine diverticulum, should be measured routinely.
\end{abstract}

"Corresponding author.

How to cite this paper: Li, C.-L., Mo, X.-T., Deng, K.-X., Chen, D.-J., Gao, X.-C., Zhai, J.-G., Liao, Y.-M., Dai, H.-R. and Cai, X. (2015) Transvaginal 3D Ultrasound Evaluation of Post-Cesarean Section Uterine Diverticulum. Open Journal of Obstetrics and Gynecology, 5, 698-702. http://dx.doi.org/10.4236/ojog.2015.512098 


\section{Keywords}

\section{Post-Cesarean Section, Uterine Diverticulum, Transvaginal 3D Ultrasound, LUS Myometrial Thickness}

\section{Introduction}

Concern is growing over the association between delivery by cesarean section and long-term maternal morbidity, as the use of cesarean section delivery has increased markedly worldwide over the last several decades [1] [2]. The long-term complications of cesarean section in relation to future reproduction have been comprehensively examined [3]. Previous studies have shown that a uterine diverticulum is present in most women with a history of cesarean section when examined by ultrasound, and this is associated with postmenstrual spotting [4].

Post-cesarean section uterine diverticulum is defined as a triangular anechoic structure at the site of the scar or a gap in the myometrium of the anterior lower uterine segment (LUS) at the site of a previous cesarean section [5]-[7]. This diverticulum, also known as a niche, is described as a defect that can be seen on an ultrasound at the site of the cesarean section scar [3]. Moreover, three-dimensional (3D) ultrasonography that enhances visualization of the LUS, myometrial thickness and the size of uterine diverticulum is a more recent tool used to diagnose post-cesarean section uterine diverticulum. In this study, we reviewed the data of 32 patients with symptomatic previous cesarean delivery scar defects who were diagnosed by 3D ultrasonography. We measured their ultrasonic LUS myometrial thickness and the size of the uterine diverticulum to assess the value of transvaginal $3 \mathrm{D}$ ultrasound in the diagnosis, treatment, and prognosis of post-cesarean section uterine diverticulum.

\section{Patients and Methods}

\subsection{Patients}

This study was conducted at the Guangzhou Institute of Obstetrics and Gynecology, the Third Affiliated Hospital of Guangzhou Medical University, Guangzhou, China. Thirty-two patients with post-cesarean section uterine diverticulum were referred to the institute from January 2012 to June 2015. The study protocol was approved by the Ethical Committee of the Guangzhou Medical University, Guangzhou, China, and all patients provided written informed consent before beginning management.

\subsection{Transvaginal 3D Ultrasound}

All transvaginal 3D sonographic examination procedures were performed and evaluated by sonologists (both board certified and with more than 15 years of obstetric and gynecologic sonography experience). Transvaginal 3D ultrasound was performed using a 7.5-MHz transducer (Voluson S8, GE Healthcare, Milwaukee, WI, USA). Patients were examined after emptying their bladders. The uterus, uterine scar, and uterine diverticulum, if present, were examined in a standardized way. The position, length, and width of the uterus and double thickness of the endometrium were registered in the midsagittal plane. A diverticulum was defined as an anechoic space with or without fluid at the presumed site of the previous cesarean section scar. When a diverticulum was identified, its length, width, and depth, as well as those of the residual myometrium at the site of the scar and the LUS myometrium, were measured in real time. In cases when more than one cesarean section scar was present the largest niche was measured.

\subsection{Data Collection}

All patients had a history of cesarean section delivery and were diagnosed with a post-cesarean section uterine diverticulum with non-specific clinical symptoms by transvaginal 3D ultrasound. The LUS myometrial thickness and size of the uterine diverticulum, which are considered to be indices of surgical options, were routinely measured. The preoperative and postoperative menstrual periods were compared and postoperative sonograms were reviewed to monitor the disappearance of the sonolucent zone in the LUS scar, as a determinant of treatment success. 


\subsection{Statistical Analysis}

The Student's t-test was used to compare the size of the uterine diverticulum before and after surgical treatment. Statistical analysis was performed using SPSS software version 18.0 (IBM, Chicago, IL, USA).

\section{Results}

Thirty-two women aged 25 - 49 years who were diagnosed with post-cesarean section uterine diverticulum were included in the study. Twenty-four patients had undergone one cesarean section delivery and eight patients had had two cesarean section deliveries. The time interval from the present post-cesarean section uterine diverticulum to the previous cesarean delivery ranged from nine months to 14 years. All the patients presented with postmenstrual spotting, and four had accompanying lower abdominal pain. The duration of their menstrual periods was $13 \pm 4.29$ days.

The transvaginal 3D ultrasounds showed a uterine diverticulum or anechoic space in the anterior part of the LUS or in the previous cesarean section scar with a thin myometrium. The mean length, width, and depth of the uterine diverticula were $18.30 \pm 2.80 \mathrm{~mm}, 9.14 \pm 3.20 \mathrm{~mm}$, and $11.49 \pm 2.71 \mathrm{~mm}$, respectively. The average LUS myometrial thickness was $3.40 \pm 0.80 \mathrm{~mm}$ (with a range from $1.6 \mathrm{~mm}$ to $6.3 \mathrm{~mm}$ ). Patients with an LUS myometrial thickness under $4 \mathrm{~mm}$ underwent resection and repair surgery (including transvaginal resection, resection and repair treatment with laparotomy, and laparoscopy combined with hysteroscopy) while hysteroscopic resections were performed for patients with an LUS myometrial thickness over $4 \mathrm{~mm}$.

After surgical treatment, two patients' sonograms still showed diverticula at the post-cesarean section scar, measuring $6 \mathrm{~mm} \times 7 \mathrm{~mm} \times 6 \mathrm{~mm}$ and $6 \mathrm{~mm} \times 8 \mathrm{~mm} \times 4 \mathrm{~mm}$. All the patients had a menstrual period lasting $6.42 \pm 1.24$ days. There were significant differences in the size of uterine diverticulum and the duration of the menstrual period after surgical treatment $(\mathrm{P}<0.05)$. The effective rate of surgery was 93.75\% (30/32) (Table 1).

Table 1. Effectiveness of surgical treatment for cesarean scar diverticulum.

\begin{tabular}{ccccc}
\hline & & \multicolumn{2}{c}{ Disappearance of sonolucent zone in the lower uterine segment scar } \\
& Menstrual period (d) & Laparotomy & $\begin{array}{c}\text { Hysteroscopy and } \\
\text { laparoscopy }\end{array}$ & Transvaginal \\
\cline { 3 - 4 } & & - & - & $100 \%(16 / 16)$ \\
Pre operation & 13.00 tionK- & $100 \%(8 / 8)$ & $100 \%(16 / 16)$ & $100 \%(8 / 8)$ \\
Post operation (3 days) & - & $100 \%(8 / 8)$ & $87.5 \%(14 / 16)$ & $100 \%(8 / 8)$ \\
Post operation (1 month) & $(6.67 \mathrm{nth}) \mathrm{K}^{*}$ & $100 \%(8 / 8)$ & $100 \%)$ \\
\hline
\end{tabular}

Note: - means the index was too insignificant to be measured; ${ }^{*} \mathrm{P}<0.01$, with remarkable difference in statistics.

\section{Discussion}

Iatrogenic diverticulum of the uterus after cesarean section is becoming increasingly common and the current rate of cesarean delivery in China is $40 \%$ to $50 \%$ [2] [8]. In a random population of women with a history of cesarean section, the prevalence of uterine diverticulum ranged from $24 \%$ to $70 \%$ and from $56 \%$ to $84 \%$ when assessed by transvaginal sonography and contrast-enhanced sonohysterography, respectively [9]. Probable risk factors are single-layer myometrium closure, multiple cesarean sections, and a retroflexed uterus. The predominant symptom related to uterine diverticulum is postmenstrual spotting [4]. Published data show no correlation between the incidence of cesarean section scar diverticulum and either the interval between first and second cesarean section or the gestational age at the time of these cesarean sections.

Interestingly, the incidence of scar diverticulum was higher in women who were older at the time of cesarean section, and it correlated positively with the number of previous cesarean sections [4] [9]. Other studies, performed in populations of women with gynecological symptoms, demonstrated that niches were significantly wider in women with postmenstrual spotting, dysmenorrhea or chronic pelvic pain, and that the prevalence of postmenstrual spotting or prolonged menstrual bleeding was higher with a larger diameter of the uterine diverticulum [9]-[11]. Symptoms in women with uterine diverticulum included dysmenorrhea (53.1\%), chronic pelvic 
pain $(36.9 \%)$, and dyspareunia (18.3\%) [9] [10].

Although there have been various treatments for CSP in recent years, no standardized diagnostic or management guidelines have been established. A scar diverticulum is easily identified using transvaginal ultrasound, and its size, shape and location can be accurately determined [4] [12] [13]. Other studies have evaluated uterine diverticulum with the use of gel instillation sonohysterography (GIS), sonohysterography (SHG) and magnetic resonance imaging (MRI). Nevertheless, transvaginal 3D ultrasonography that enhances visualization of the LUS, myometrial thickness, and the length, width and depth of the uterine diverticulum has been more recently used for accurate diagnosis of post-cesarean section uterine diverticulum [14].

Surgical approaches to treating cesarean section scar abnormalities include hysteroscopic techniques, laparotomy and defect repair, and hysterectomy. Complete hysterectomy is curative in patients who do not wish to have more children [15] [16]. From the published data, the treatment of patients with post-cesarean section uterine diverticulum uses $4 \mathrm{~mm}$ as a threshold LUS myometrial thickness. Resection and repair surgery includes transvaginal resection, resection, and repair treatment with laparotomy and laparoscopy combined with hysteroscopy.

In our study, all 32 patients were diagnosed with post-cesarean section uterine diverticulum by transvaginal 3D ultrasound. Resection and repair surgery was recommended for patients with an LUS myometrial thickness under $4 \mathrm{~mm}$ to prevent surgical complications such as hemorrhage and uterine rupture. Hysteroscopic resection was recommended for patients with an LUS myometrial thickness over $4 \mathrm{~mm}$ as it is minimally invasive and there is less blood loss. Transvaginal 3D ultrasound was used to monitor the disappearance of sonolucent zone in the lower uterine segment scar three days, one month and three months after surgery.

\section{Conclusion}

In conclusion, transvaginal 3D ultrasound is an accurate method for detecting post-cesarean section uterine diverticulum and is helpful for assessing surgical options and prognosis. The LUS myometrial thickness, which is considered as an optional index of post-cesarean section uterine diverticulum, should be measured routinely. However, comparative studies of different therapies with sufficient methodological quality are lacking. Future studies involving sufficient sample size, long-term follow-up, and validated instruments are needed.

\section{Acknowledgements}

The authors thank Oxford Language Editing for helping us to edit and proofread this manuscript.

\section{Funding}

Support for this research was provided by the Clinical research about Cesarean scar pregnancy from precious cesarean delivery of Science and Technology Innovation Project from Guangzhou Medical University (2014); to explore the application of Early clinical training and early research training education mode in lower grade MBBS student of Guangzhou Medical University, the research on practical teaching of the "Twelfth Five Year Plan" for education science (Key Funding 2014); The practice and Research of the implementation of Double Early Education, Early Clinical Training \& Early Research Training, for medical undergraduates, Research on the teaching reform of clinical teaching base in Guangdong Province in 2014, key Funding in Teaching Research, Funding number 2014JDA028; the Prevention and Control of Major Obstetric Disease major collaborative innovation project of the Educational Bureau of Guangzhou City (medical and health grant no. 13xt04, 2013), and the Collaborative Innovation Centre for Prevention and Control of Major Obstetric Disease collaborative innovation platform of the educational and financial departments of Guangdong Province (regional development grant, 2014).

\section{Conflicts of Interest}

The authors have no conflicts of interest to declare in relation to this article.

\section{References}

[1] Betran, A.P., Merialdi, M., Lauer, J.A., et al. (2007) Rates of Caesarean Section: Analysis of Global, Regional and Na- 
tional Estimates. Paediatric and Perinatal Epidemiology, 21, 98-113. http://dx.doi.org/10.1111/j.1365-3016.2007.00786.x

[2] Luo, L., Niu, G., Wang, Q., et al. (2012) Vaginal Repair of Cesarean Section Scar Diverticula. Journal of Minimally Invasive Gynecology, 19, 454-458. http://dx.doi.org/10.1016/j.jmig.2012.03.012

[3] van der Voet, L.F., Bij de Vaate, A.M., Veersema, S., et al. (2014) Long-Term Complications of Caesarean Section. The Niche in the Scar: A Prospective Cohort Study on Niche Prevalence and Its Relation to Abnormal Uterine Bleeding. BJOG, 121, 236-244. http://dx.doi.org/10.1111/1471-0528.12542

[4] Bij de Vaate, A.J., Brölmann, H.A., van der Voet, L.F., et al. (2011) Ultrasound Evaluation of the Cesarean Scar: Relation between a Niche and Postmenstrual Spotting. Ultrasound in Obstetrics \& Gynecology, 37, 93-99. http://dx.doi.org/10.1002/uog.8864

[5] Monteagudo, A., Carreno, C. and Timor-Tritsch, I.J. (2001) Saline Infusion Sonohysterography in Nonpregnant Women with Previous Caesarean Delivery: The "Niche" in the Scar. Journal of Ultrasound in Medicine, 20, 1105-1115.

[6] Thurmond, A.S., Harvey, W.J. and Smith, S.A. (1999) Cesarean Section Scar as a Cause of Abnormal Vaginal Bleeding: Diagnosis by Sonohysterography. Journal of Ultrasound in Medicine, 18, 13-16.

[7] Wu, Q.F., Nie, L. and Zhang, Y. (2011) Clinical Analysis on IVF-ET Treatment of 9 Cases of Post-Cesarean Section Uterine Diverticulum. Journal of Reproduction \& Contraception, 22, 183-190. http://dx.doi.org/10.1016/S1001-7844(12)60014-5

[8] Li, N., Wang, Y., Wang, B., et al. (2007) The Partial Survey of Cesarean Section in Hospitals and Health Care Unit in China. Maternal and Child Health in China, 22, 3305-3307.

[9] Bij de Vaate, A.J., van der Voet, L.F., Naji, O., et al. (2014) Prevalence, Potential Risk Factors for Development and Symptoms Related to the Presence of Uterine Niches Following Cesarean Section: Systematic Review. Ultrasound in Obstetrics \& Gynecology, 43, 372-382. http://dx.doi.org/10.1002/uog.13199

[10] Wang, C.B., Chiu, W.W., Lee, C.Y., et al. (2009) Cesarean Scar Defect: Correlation between Cesarean Section Number, Defect Size, Clinical Symptoms and Uterine Position. Ultrasound in Obstetrics \& Gynecology, 34, 85-89. http://dx.doi.org/10.1002/uog.6405

[11] Uppal, T., Lanzarone, V. and Mongelli, M. (2011) Sonographically Detected Caesarean Section Scar Defects and Menstrual Irregularity. Journal of Obstetrics and Gynaecology, 31, 413-416. http://dx.doi.org/10.3109/01443615.2011.577252

[12] Rajiah, P., Eastwood, K.L., Gunn, M.L., et al. (2009) Uterine Diverticulum. Obstetrics \& Gynecology, 113, $525-527$. http://dx.doi.org/10.1097/AOG.0b013e31818da0b9

[13] Osser, O.V., Jokubkiene, L. and Valentin, L. (2009) High Prevalence of Defects in Cesarean Section Scars at Transvaginal Ultrasound Examination. Ultrasound in Obstetrics \& Gynecology, 34, 90-97. http://dx.doi.org/10.1002/uog.6395

[14] Gizzo, S., Zambon, A., Saccardi, C., et al. (2013) Effective Anatomical and Functional Status of the Lower Uterine Segment at Term: Estimating the Risk of Uterine Dehiscence by Ultrasound. Fertility and Sterility, 99, 496-501. http://dx.doi.org/10.1016/i.fertnstert.2012.10.019

[15] Erickson, S.S. and Van Voorhis, B.J. (1999) Intermenstrual Bleeding Secondary to Cesarean Scar Diverticuli: Report of Three Cases. Obstetrics \& Gynecology, 93, 802-805. http://dx.doi.org/10.1016/S0029-7844(98)00314-7

[16] Evan, P. and Kramer, B. (2008) Uterine Diverticula and Accessory Ducts. AJR, 19, W71. http://dx.doi.org/10.2214/AJR.07.3679 